\title{
Non-Singular String-Cosmologies From Exact Conformal Field Theories
}

\author{
H.J. de Vega ${ }^{\mathrm{a}}$, $\underline{\text { A.L. Larsen }}^{\mathrm{b}}$ and N. Sánchez ${ }^{\mathrm{c}}$ \\ ${ }^{a}$ Université de Paris VI et VII. 4, Place Jussieu, 75252 Paris, France. \\ ${ }^{\text {b} D e p a r t m e n t ~ o f ~ P h y s i c s, ~ U n i v e r s i t y ~ o f ~ O d e n s e, ~ C a m p u s v e j ~ 55, ~} 5230$ Odense M, Denmark. \\ ${ }^{\mathrm{c}}$ Observatoire de Paris, DEMIRM. 61, Avenue de l'Observatoire, 75014 Paris, France.
}

\begin{abstract}
Non-singular two and three dimensional string cosmologies are constructed using the exact conformal field theories corresponding to $\mathrm{SO}(2,1) / \mathrm{SO}(1,1)$ and $\mathrm{SO}(2,2) / \mathrm{SO}(2,1)$. All semi-classical curvature singularities are canceled in the exact theories for both of these cosets, but some new quantum curvature singularities emerge. However, considering different patches of the global manifolds, allows the construction of non-singular spacetimes with cosmological interpretation. In both two and three dimensions, we construct non-singular oscillating cosmologies, non-singular expanding and inflationary cosmologies including a de Sitter (exponential) stage with positive scalar curvature as well as non-singular contracting and deflationary cosmologies. Similarities between the two and three dimensional cases suggest a general picture for higher dimensional coset cosmologies: Anisotropy seems to be a generic unavoidable feature, cosmological singularities are generically avoided and it is possible to construct non-singular cosmologies where some spatial dimensions are experiencing inflation while the others experience deflation.
\end{abstract}

\section{INTRODUCTION}

Fundamental quantum strings demand a conformally invariant background for quantum consistency (conformal invariance is a necessary although not sufficient condition for consistency). However, most curved spacetimes that were historically of physical interest in general relativity and cosmology are not conformally invariant. For instance black hole spacetimes and FriedmanRobertson-Walker universes, are not of this type. In string theory, a large amount of generalizations of these general relativity solutions have been obtained as solutions of the renormalization group $\beta$-function equations (low energy effective string equations). These solutions involve, besides the metric, a number of massless fields including the dilaton and antisymmetric tensor fields. The $\beta$ function equations are the basis for most investigations in string cosmology. However, they are perturbative and only known to the lowest orders in $\alpha^{\prime}$, and therefore, the corresponding solutions are only ensured to be conformally invariant to the lowest orders in $\alpha^{\prime}$. It is not generally clear how the higher order corrections will change these solutions, and possible non-perturbative solutions seem to be completely missed in this framework.

A different approach to string cosmology is based on group manifolds and coset spaces and the corresponding WZW [1] and gauged WZW models [2]. These models provide new curved backgrounds that are conformally invariant to all orders in $1 / k$ (where $k \sim 1 / \alpha^{\prime}$ is the level of the WZW model). In the case of group manifolds, these models are generally too simple to describe physically interesting and realistic cosmologies. This is exemplified by the well-studied $S L(2, R)$ WZW model, which describes Anti-de Sitter space. For the coset spaces, on the other hand, the models are generally so complicated that it is difficult even to extract what they describe from a manifest spacetime point of view. In fact, the exact (all orders in $1 / k$ ) metric, dilaton etc, have only been obtained in a limited number of low-dimensional cases (for a rewiew of such solutions, see Refs. 344). And in most of these cases, the physical spacetime properties have not really been extracted so far. Most studies of coset space gauged WZW models still restrict themselves to lowest order in $1 / k$, and are thus es- 
sentially equivalent to the studies based on the $\beta$-function equations.

The purpose of the present paper is to show that interesting non-trivial and non-singular cosmologies can be obtained from the cosets $S O(2,1) / S O(1,1)$ and $S O(2,2) / S O(2,1)$. Various aspects of string theory on these cosets have been investigated in the literature, see for instance Refs. [1]- [10]. Cosmological interpretations have been considered in Refs. [9, 10, 12, but mostly to lowest order in $1 / k$

The two dimensional coset $S O(2,1) / S O(1,1)$, to all orders in $1 / k$, has been given a cosmological interpretation in 10], but only in one of the coordinate patches.

As for the three dimensional coset $S O(2,2) / S O(2,1)$, a cosmological interpretation was attempted in [9], but only in certain coordinate patches and only to first order in $1 / k$. The cosmologies obtained in [9], however, are completely changed in the exact theory.

Interestingly enough, quantum corrections not only cancel the semiclassical singularities but also change the sign of the scalar curvature for oscillatory cosmologies. In non-oscillating cosmologies, the scalar curvature evolves from positive for large and negatives times $t$, to negative near $t=0$.

\section{TWO-DIMENSIONAL COSET}

The $S O(2,1) / S O(1,1)$ metric and dilaton, to all orders in $1 / k$, are given by [6]:

$$
\begin{aligned}
& d s^{2}=2(k-2)\left[\frac{d b^{2}}{4\left(b^{2}-1\right)}-\beta(b) \frac{b-1}{b+1} d x^{2}\right] \\
& \Phi(b)=\ln \frac{b+1}{\sqrt{\beta(b)}}+\text { const. }
\end{aligned}
$$

where:

$\beta(b) \equiv\left(1-\frac{2}{k} \frac{b-1}{b+1}\right)^{-1}$

In these $(b, x)$-coordinates, the global manifold is

$-\infty<b<\infty, \quad-\infty<x<\infty$.

The scalar curvature is given by:

$R(b)=\frac{4}{k-2} \frac{k(k-4)+k(k-2) b}{[k+2+(k-2) b]^{2}}$
We see here the presence of new quantum curvature singularities at

$b=-\frac{k+2}{k-2}$

plus coordinate singularities (horizons) at $b= \pm 1$.

In the semi-classical limit $(k \rightarrow \infty)$, the scalar curvature reduces to

$R(b) \stackrel{k \rightarrow \infty}{=} \frac{4}{k} \frac{1}{1+b}$

Comparison of (4) and (6) shows that in the semiclassical limit the quantum curvature singularities coalesce with the coordinate singularities (horizons) at $b= \pm 1$.

The fact that the semi-classical curvature singularities become reduced to coordinate singularities, and that new curvature singularities appear in the exact (all orders in $1 / k$ ) theory, will turn out to hold also for the 3 -dimensional cosets, to be discussed in the next section.

For conformal invariance we demand that

$C\left(\frac{S O(2,1)}{S O(1,1)}\right)=26$

leading to $k=9 / 4$. Thus the curvature singularity (5) is located at $b=-17$, that is to say, in the coordinate patch to the left of the left horizon in the $(b-x)$-coordinate system. Clearly, it is then possible to construct non-singular spacetimes by considering the other coordinate patches. In the present case, we are interested in constructing simple cosmological spacetimes, by which we mean, spacetimes with signature $(-+)$ and lineelement of the form

$d s^{2}=-d t^{2}+A^{2}(t) d x^{2}$

where $t$ plays the role of cosmic time, $x$ is the spatial coordinate and $A(t)$ is the scale factor.

\subsection{Oscillating Cosmology}

Considering the coordinate patch between the two horizons, $|b| \leq 1$, and using the parametrization $b=\cos 2 t$, we get the cosmology [10]

$d s^{2}=\frac{1}{2}\left(-d t^{2}+\frac{\tan ^{2} t}{1+\frac{8}{9} \tan ^{2} t} d x^{2}\right)$

$\Phi(t)=\ln \left(\cos ^{2} t \sqrt{1+\frac{8}{9} \tan ^{2} t}\right)+$ const. 
The scalar curvature

$$
R(t)=-72 \frac{4-\cos ^{2} t}{\left(8+\cos ^{2} t\right)^{2}}
$$

is oscillating, finite and always negative

$$
\begin{aligned}
R(t) \in\left[-\frac{9}{2},-\frac{8}{3}\right] \\
<R(t)>=-\frac{5}{\sqrt{2}} \approx-3.53 . .
\end{aligned}
$$

It is an interesting observation that the quantum corrections not only cancel the semi-classical curvature singularities, but also change the sign of the scalar curvature, since semi-classically $(k \rightarrow$ $\infty)$

$$
R(t) \rightarrow \frac{4}{k} \frac{1}{1+\cos 2 t}
$$

which is always positive (and sometimes positive infinity). We shall see in the next section that this feature also holds in the 3-dimensional case.

Finally, we make a few comments about the dilaton (10). In the present notation and conventions, the string-coupling $g_{s}$ is given by

$g_{s}=e^{-\Phi / 2}=\left(\cos ^{2} t \sqrt{1+\frac{8}{9} \tan ^{2} t}\right)^{-1 / 2}$

up to an arbitrary positive multiplicative constant. It follows that we should strictly speaking only trust the cosmological solution in the regions near $t=0, t= \pm \pi, t= \pm 2 \pi$ etc. That is to say, in the regions where the scale factor is small. In the other regions where the scale factor is large, we should expect that string-loop corrections will be important and possibly change the solution dramatically. Thus, concentrating on (say) the region around $t=0$, the line-element (9) describes a universe experiencing deflationary contraction (negative $t$ ) followed by deflationary expansion (positive $t$ ). And always with negative scalar curvature.

\subsection{Deflationary Cosmology}

The oscillating cosmology (9) was obtained for the value $k=9 / 4$, corresponding to conformal invariance. However, using other values of $k$, it is possible to construct other types of non-singular cosmologies. In that case, conformal invariance must be ensured by adding other conformal field theories. Moreover, problems with unitarity will possibly appear in such cases. In this subsection, we shall not deal with these problems, but instead concentrate on the possible cosmologies that can be obtained by keeping $k$ arbitrary.

Taking $k=-|k|<0$, we observe that the curvature singularity (5) is located between the two horizons $b= \pm 1$. It is then possible to construct non-singular spacetimes by specializing to the two patches outside the horizons.

Consider first the patch $b \geq 1$ using the parametrization $b=\cosh 2 t$. Then Eqs.(1)-(3) become

$d s^{2}=2(2+|k|)\left(-d t^{2}+\frac{\tanh ^{2} t}{1+\frac{2}{|k|} \tanh ^{2} t} d x^{2}\right)$

$\Phi(t)=\ln \left(\cosh ^{2} t \sqrt{1+\frac{2}{|k|} \tanh ^{2} t}\right)+$ const. $(16)$

Notice that it is crucial to include the overall prefactor $2(k-2)$ in the line-element to get the desired signature.

In this case the scalar curvature is given by

$R(t)=-\frac{2|k|}{2+|k|} \frac{(2+|k|) \cosh ^{2} t+1}{\left[(2+|k|) \cosh ^{2} t-2\right]^{2}}$

while the Hubble function and its derivative become

$H=\frac{\dot{A}(t)}{A(t)}=\frac{\cosh t}{\left(\cosh ^{2} t+\frac{2}{|k|} \sinh ^{2} t\right) \sinh t}$

$\dot{H}=-|k| \frac{(2+|k|)\left(1+2 \sinh ^{2} t\right) \cosh ^{2} t-2}{\left[(2+|k|) \cosh ^{2} t-2\right]^{2} \sinh ^{2} t}$

It follows that the universe has negative scalar curvature and it is always deflationary $(\dot{H}<$ $0)$. More precisely, starting from flat Minkowski space $(t=-\infty)$, the universe experiences deflationary contraction until $t=0$. Still deflationary, it then expands and approaches flat Minkowski space again for $t \rightarrow \infty$; see Figure 1 .

The string-coupling in this case is given by

$g_{s}=e^{-\Phi / 2}=\left(\cosh ^{2} t \sqrt{1+\frac{2}{|k|} \tanh ^{2} t}\right)^{-1 / 2}$ 


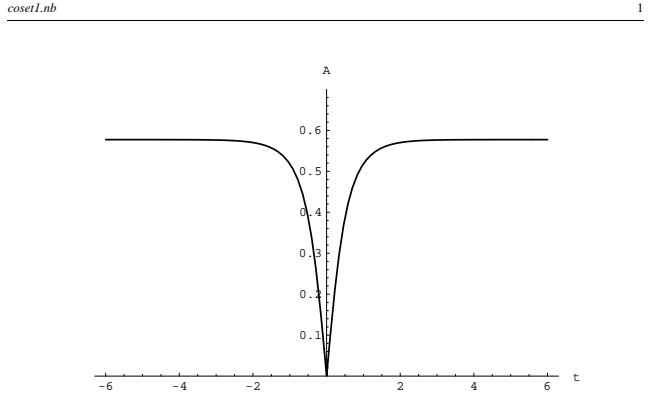

Figure 1. The scale factor (15) as a function of time. Here shown for $k=-1$. This cosmology is always deflationary.

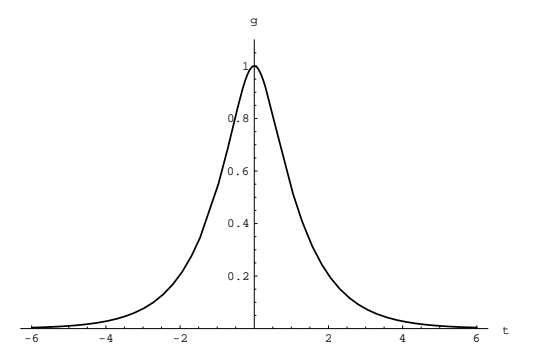

Figure 2. The string-coupling (20) corresponding to Figure 1. The string-coupling is finite for all $t$.

which is always finite, and in fact goes to zero for $t \rightarrow \pm \infty$; see Figure 2. Thus, this non-singular cosmology should be trusted everywhere.

\subsection{Inflationary Cosmology}

A more interesting cosmology can be constructed by considering the patch $b \leq-1$ (and still $k<0$ ) instead of $b \geq 1$. Using the parametrization $b=-\cosh 2 t$, Eqs.(1)-(3) become

$d s^{2}=2(2+|k|)\left(-d t^{2}+\frac{\operatorname{coth}^{2} t}{1+\frac{2}{|k|} \operatorname{coth}^{2} t} d x^{2}\right)$

$$
\Phi(t)=\ln \left(\sinh ^{2} t \sqrt{1+\frac{2}{|k|} \operatorname{coth}^{2} t}\right)+\text { const. }
$$

The scalar curvature is now given by

$$
R(t)=\frac{2|k|}{2+|k|} \frac{(2+|k|) \sinh ^{2} t-1}{\left[(2+|k|) \sinh ^{2} t+2\right]^{2}}
$$

while the Hubble function and its derivative become

$$
\begin{aligned}
& H=-\frac{\sinh t}{\left(\sinh ^{2} t+\frac{2}{|k|} \cosh ^{2} t\right) \cosh t} \\
& \dot{H}=|k| \frac{(2+|k|)\left(1+2 \sinh ^{2} t\right) \sinh ^{2} t-2}{\left[(2+|k|) \sinh ^{2} t+2\right]^{2} \cosh ^{2} t}
\end{aligned}
$$

Now the situation is a little more complicated than in the $b \geq 1$ patch. However, notice that

$$
R(t)>0 \quad \Leftrightarrow \quad \cosh ^{2} t>\frac{3+|k|}{2+|k|}
$$

as well as

$$
\dot{H}>0 \Leftrightarrow \cosh ^{2} t>\frac{1}{4}\left(3+\sqrt{1+\frac{16}{2+|k|}}\right)
$$

Thus the universe starts out as flat Minkowski space at $t=-\infty$. It then experiences inflationary $(\dot{H}>0)$ expansion with positive scalar curvature. Just before the scale factor reaches its maximal value at $t=0$, the scalar curvature becomes negative and the expansion becomes deflationary. For $t>0$, the evolution is simply time-reversed; See Figure 3 .

It is particularly interesting that the universe is inflationary $(\dot{H}>0)$ with positive scalar curvature for (large) negative $t$. This suggests that the universe goes through some kind of de Sitter phase. This is confirmed by expanding the scale factor for large negative $t(t<<0)$

$$
\begin{aligned}
A(t) & =\sqrt{\frac{\operatorname{coth}^{2} t}{1+\frac{2}{|k|} \operatorname{coth}^{2} t}} \\
& \approx \sqrt{\frac{|k|}{2+|k|}}\left(1+\frac{2|k|}{2+|k|} e^{2 t}\right)
\end{aligned}
$$

thus there is in fact an element of exponential expansion. 


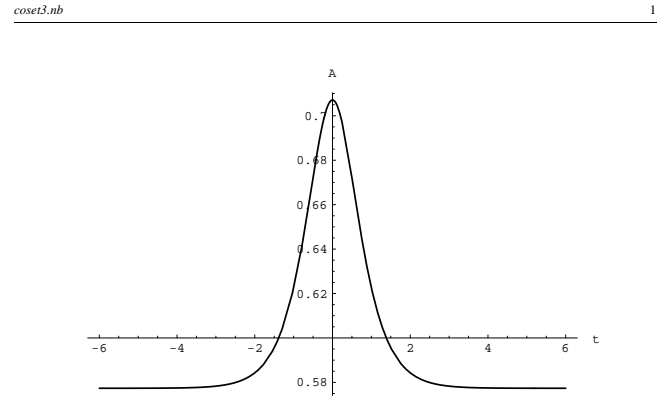

Figure 3. The scale factor (21) as a function of time. Here shown for $k=-1$. This cosmology is expanding and inflationary for large negative $t$.

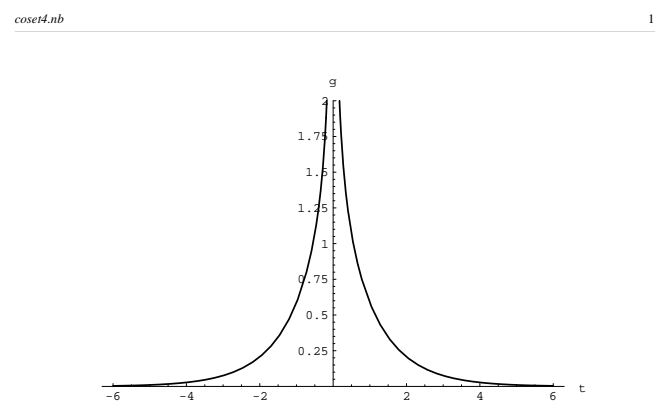

Figure 4. The string-coupling (30) corresponding to Figure 3. The string-coupling is finite everywhere except near $t=0$.

Finally, let us consider the string-coupling also in this case. It is given by

$g_{s}=e^{-\Phi / 2}=\left(\sinh ^{2} t \sqrt{1+\frac{2}{|k|} \operatorname{coth}^{2} t}\right)^{-1 / 2}$

which is finite everywhere except near $t=0$; see Figure 4. In particular, the string-coupling is finite during the phase of inflationary expansion.

\section{THREE-DIMENSIONAL COSET}

The computations are now going to be somewhat more complicated. Here we shall only present the results; the detailed computations can be found in 13 .

The $S O(2,2) / S O(2,1)$ metric and dilaton, to all orders in $1 / k$, are given by 8$]$

$$
\begin{aligned}
& d s^{2}=2(k-2)\left[\frac{d b^{2}}{4\left(b^{2}-1\right)}+g_{u u} d u^{2}+g_{v v} d v^{2}\right. \\
& \left.+2 g_{u v} d u d v\right] \\
& \Phi(b, u, v)=\ln \frac{\left(b^{2}-1\right)(v-u-2)}{\sqrt{\beta(b, u, v)}}+\text { const. }
\end{aligned}
$$

where the explicit expressions for $g_{u u}, g_{v v}, g_{u v}$ and $\beta$ can be found in [8].

In these $(b, u, v)$-coordinates, the global manifold for $S O(2,2) / S O(2,1)$ is [7, 8]

$$
\begin{array}{ll}
b^{2}>1 & \text { and } \quad u v>0, \\
b^{2}<1 & \text { and } \quad u v<0,
\end{array}
$$

excluding $0<v<u+2<2$ in the second case. However, other regions in the $(b, u, v)$ space can be reached by going for instance to the $S O(3,1) / S O(2,1)$ coset, for which the metric and dilaton are formally the same as above. For more details, see Refs. [7, 8].

The general expression for the scalar curvature, corresponding to the metric (31), is quite complicated. It is most conveniently written as a fourth order polynomium in $b$ divided by the square of a second order polynomium in $b$ :

$R=\frac{4}{k-2} \frac{c_{0}+c_{1} b+c_{2} b^{2}+c_{3} b^{3}+c_{4} b^{4}}{\left(d_{0}+d_{1} b+d_{2} b^{2}\right)^{2}}$

where the coefficients $c_{i}$ and $d_{j}$ are functions of the remaining coordinates $(u, v)$ and the parameter $k$

$$
\begin{aligned}
c_{i} & =c_{i}(u, v ; k), \quad i=0,1,2,3,4 \\
d_{j} & =d_{j}(u, v ; k), \quad j=0,1,2
\end{aligned}
$$

Their explicit expressions are given in [13].

Comparison of the semi-classical expression $(k \rightarrow \infty)$ and the exact expression (33) for the scalar curvature shows that all semi-classical curvature singularities $(b=1, b=-1$ and $v=u+2)$ have disappeared, but that new quantum curvature singularities have appeared. The situation is thus completely analogous to the 2-dimensional 
case: The semi-classical curvature singularities become coordinate singularities (horizons), but new quantum curvature singularities have appeared elsewhere. The curvature singularities in the exact geometry correspond to the zeroes of the denominator in (33). They precisely correspond to the singularities of the function $\beta(b, u, v)$.

By considering different values of $k$, one can move the singularity surface around in the global manifold. It is then possible, as in the 2-dimensional case, to construct non-singular spacetimes by considering a single coordinate patch. Notice that for conformal invariance, we should demand that

$C\left(\frac{S O(2,2)}{S O(2,1)}\right)=26$

leading to $k=(39 \pm 5 \sqrt{13}) / 23$.

\subsection{Oscillating Cosmology}

Consider first the case where $k=(39+$ $5 \sqrt{13}) / 23 \approx 2.48 \ldots$. It is then possible to construct a cosmology in the coordinate patch

$|b| \leq 1, \quad u \geq 0, \quad v \leq 0$

using the parametrization

$b=\cos 2 t, \quad u=2 x^{2}, \quad v=-2 y^{2}$

It is easy to see that this is a non-singular cosmology with $t$ playing the role of cosmic time, $(x, y)$ are the spatial coordinates and the signature is $(-++)$, as it should be. The cosmology is however somewhat complicated: It is periodic in time, but non-homogeneous and highly nonisotropic. In fact, the 'scale factors' for the two spatial directions are oscillating with a phase difference of $\pi / 2$.

To gain a little more insight into this cosmology, we consider the region near the spatial 'origin' $(x, y)=(0,0)$. The scalar curvature reduces to

$R(t, 0,0)=\frac{1}{k-2} \frac{\tilde{c}_{0}+\tilde{c}_{2} \cos ^{2} 2 t+\tilde{c}_{4} \cos ^{4} 2 t}{\left[k^{2}-(k-2)^{2} \cos ^{2} 2 t\right]^{2}}$

where the coefficients $\left(\tilde{c}_{0}, \tilde{c}_{2}, \tilde{c}_{4}\right)$ are given in 13 . It follows that

$R(t, 0,0) \in[-3.1435 . .,-2.2988 .$. as well as

$$
<R(t, 0,0)>\approx-2.7126 \text {. }
$$

where the numerical values were obtained for $k=(39+5 \sqrt{13}) / 23$. As in the 2-dimensional case, we notice therefore that the quantum corrections changed the sign of the curvature, since semi-classically $(k \rightarrow \infty)$

$R(t, 0,0) \stackrel{k \rightarrow \infty}{=} \frac{4}{k} \frac{3+\cos ^{2} t}{2 \sin ^{2} t}$

which is always positive (and sometimes positive infinity).

In the region near $(x, y)=(0,0)$, one can further define scale factors

$$
\begin{aligned}
& A(t)=\sqrt{g_{x x}(t, 0,0)} \\
& B(t)=\sqrt{g_{y y}(t, 0,0)}
\end{aligned}
$$

both of which oscillate between 0 and $\sqrt{k-1} \approx$ 1.216.. with a phase difference of $\pi / 2$.

Finally, let us return to the dilaton (for generic $(t, x, y))$. The string-coupling is given by

$g_{s}=\left[\frac{\left(x^{2}+y^{2}+1\right) \cos ^{2} t \sin ^{2} t}{\sqrt{\beta(t, x, y)}}\right]^{-1 / 2}$

Thus the string-coupling blows up at $t=0$, $t= \pm \pi / 2, t= \pm \pi$ etc. It means that we should only trust the solution in the intermediate regions where $g_{s}$ is not large.

\subsection{Non-Oscillating Cosmologies}

In the previous subsection we obtained an oscillating cosmology using the value $k=(39+$ $5 \sqrt{13}) / 23 \approx 2.48$.., corresponding to conformal invariance. Most formulas were however presented keeping $k$ arbitrary, and the oscillating cosmologies in fact exist for arbitrary $k>2$. In this subsection we shall show that it is possible to construct non-singular 3-dimensional nonoscillating cosmologies when $k<1$. Interestingly enough, the condition (34) of conformal invariance gives rise to the possibility $k=(39-$ $5 \sqrt{13}) / 23 \approx 0.912 .$. , but in the following we just keep $k$ arbitrary but less than 1 . Possible problems with unitarity will not be dealt with here. 
Thus we take $k<1$ and consider first the patch

$b \geq 1, \quad u \geq 0, \quad v \leq 0$

and use the parametrization

$b=\cosh 2 t, \quad u=2 x^{2}, \quad v=-2 y^{2}$

Actually the patch (44) is not part of the global manifold for $S O(2,2) / S O(2,1)$, so one has to go to the de Sitter coset $S O(3,1) / S O(2,1)$ instead [7,8]. The general expressions for the metric and dilaton (31)-(32) are however unchanged.

Using the parametrization (45), the new metric and dilaton are similar to the ones of Subsection 3.1. except that trigonometric functions become hyperbolic functions and $k-1$ becomes $1-k$. Since we now consider the case where $k<1$, it is then clear that it describes a cosmology with the correct signature $(-++)$. Furthermore, it is easily seen that the cosmology is non-singular. The change from trigonometric functions to hyperbolic functions obviously has dramatic consequences. The cosmology is however still non-isotropic and non-homogeneous, but it is no longer oscillating. In fact, the timedependence of the metric actually disappears for $t \rightarrow \pm \infty$, i.e., the universe becomes static in these limits (however, the dilaton stays timedependent). More precisely, both 'scale factors' start out with constant values at $t=-\infty$. Then one of them increases monotonically towards a maximal value, while the other one decreases monotonically to zero for $t \rightarrow 0_{-}$. For $t>0$, their behaviour is simply time-reversed.

As for the oscillating cosmology in the previous subsection, let us define scale factors analogous to (41)-(42). In the present case the results are shown in Figure 5. A careful analysis of the corresponding Hubble functions and their derivatives shows that for $t<<0$, one scale factor is contracting and deflationary while the other scale factor is expanding and inflationary. This is most easily seen from the expansions

$$
\begin{aligned}
& A(t) \approx \sqrt{\frac{1-k}{2-k}}\left(1-2 \frac{1-k}{2-k} e^{2 t}\right), t<<0 \\
& B(t) \approx \sqrt{\frac{1-k}{2-k}}\left(1+2 \frac{1-k}{2-k} e^{2 t}\right), t<<0
\end{aligned}
$$

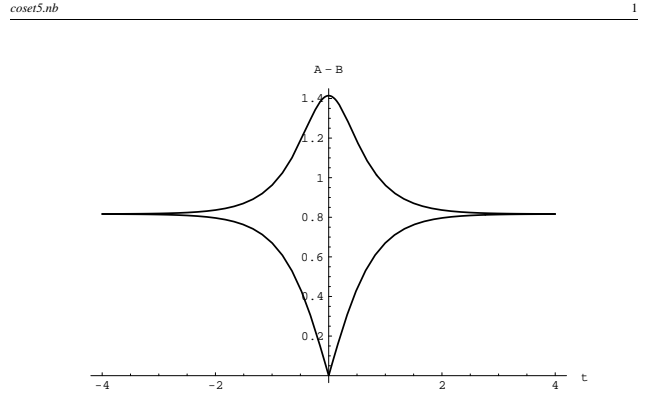

Figure 5. The two scale factors of Subsection 3.2. as a function of time. Here shown for $k=-1$. For large negative $t$, one scale factor is expanding and inflationary while the other scale factor is contracting and deflationary.

and still taking into account that $k<1$.

It is interesting that the scale factor $A(t)$ is very similar to the scale factor of the deflationary 2-dimensional cosmology of Subsection 2.2, while the scale factor $B(t)$ is very similar to the scale factor of the inflationary 2-dimensional cosmology of Subsection 2.3. In particular, the scale factor $B(t)$ has en element of exponential expansion as seen from Eq.(47). In that sense, the 2dimensional coset comprises all the main features of the 3-dimensional coset.

The string-coupling in this case is shown in Figure 6. It is finite everywhere except near $t=0$. Asymptotically $(t \rightarrow \pm \infty)$ it goes to zero. Thus the solution should be trusted everywhere except near $t=0$.

It is also possible to construct a non-singular non-oscillating cosmology in the patch

$b \leq-1, \quad u \geq 0, \quad v \leq 0$

using the parametrization

$b=-\cosh 2 t, \quad u=2 x^{2}, \quad v=-2 y^{2}$

and still taking $k<1$ (also in this case one has to go to the de Sitter coset $S O(3,1) / S O(2,2)$, see Ref. [7, \#)). However, the resulting cosmology is identical with the previous one, up to an interchange of $x$ and $y$. 


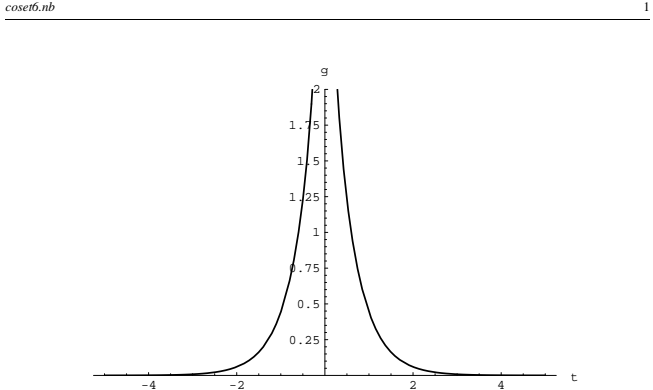

Figure 6. The string-coupling corresponding to Figure 5. The string-coupling is finite everywhere except near $t=0$.

\section{CONCLUSIONS}

The cosmological geometries found in this paper are exact conformal field theories. That is, they describe string vacua. Physically, these are spacetimes where the (massless) dilaton and graviton fields are present. We find that such manifolds provide non-singular spacetimes with cosmological interpretation. These are of two types: oscillating and non-oscillating cosmologies. The non-oscillating metrics start as Minkowski spacetimes for early times, evolve through inflationary expansion (with positive scalar curvature), then pass through a deflationary contraction ending as a Minkowski spacetime for late times.

The coset string cosmologies studied in this paper correspond to analogies of de Sitter spacetime in string vacua. In General Relativity, the global de Sitter manifold describes a contracting phase (for $t \leq 0$ ) and then an expanding universe for $t \geq 0$. Only the expanding patch describes the physical space. Similarly, in string cosmologies one should not consider the physical space as the whole global manifold but only a part of it.

\section{REFERENCES}

1. E. Witten, Commun. Math. Phys. 92 (1984) 455.

2. E. Witten, Phys. Rev. D44 (1991) 314.
3. I. Bars in String Quantum Gravity and Physics at the Planck Scale, Proceedings of the Erice Workshop held in June 1992. Edited by N. Sánchez, World Scientific, 1993, p. 224.

4. A. Tseytlin, Class. Quantum Grav. 12 (1995) 2365 .

5. I. Bars and K. Sfetsos, Mod. Phys. Lett. A7 (1992) 1091.

6. R. Dijkgraaf, H. Verlinde and E. Verlinde, Nucl. Phys. B371 (1992) 269.

7. I. Bars and K. Sfetsos, Phys. Rev. D46 (1992) 4495.

8. I. Bars and K. Sfetsos, Phys. Rev. D46 (1992) 4510 .

9. E.S. Fradkin and V.Y. Linetsky, Phys. Lett. B277 (1992) 73.

10. A. Tseytlin and C. Vafa, Nucl. Phys. B372 (1992) 443.

11. L. Dixon, J. Lykken and M. Peskin, Nucl. Phys. B325 (1989) 329. I. Bars, Nucl. Phys. B334 (1990) 125. I. Bars and D. Nemeschansky, Nucl. Phys. B348 (1991) 89. E.S. Fradkin and V.Y. Linetsky, Phys. Lett. B261 (1991) 26. M. Crescimanno, Mod. Phys. Lett. A7 (1992) 489. I. Bars and K. Sfetsos, Phys. Lett. B277 (1992) 269. K. Sfetsos, Nucl. Phys. B389 (1993) 424. K. Sfetsos and A. Tseytlin, Phys. Rev. D49 (1994) 2933. A.H. Chamseddine, Phys. Lett. B275 (1992) 63. P. Ginsparg and F. Quevedo, Nucl. Phys. B385 (1992) 527. M.J. Perry and E. Teo, Phys. Rev. Lett. 70 (1993) 2669. I. Bars and K. Sfetsos, Phys. Rev. D48 (1993) 844. I. Bars and K. Sfetsos, Phys. Lett. B301 (1993) 183. D. Gershon, Phys. Rev. D50 (1994) 6481. D. Gershon, Phys. Rev. D51 (1995) 4387. I. Bakas and K. Sfetsos, Phys. Rev. D54 (1996) 3995.

12. C. Kounnas, D. Lüst, Phys. Lett. B289 (1992) 56. C. Nappi and E. Witten, Phys. Lett. B293 (1992) 309. L.A. Pando Zayas, Phys. Lett. B407 (1997) 121. L.A. Pando Zayas, Phys. Lett. B398 (1997) 274.

13. H.J. de Vega, A. L. Larsen and N. Sánchez, Phys. Rev. D 61, 066003 (2000). 